\title{
Decentralized optimization of the flexible production lines
}

\author{
Malega, P. ${ }^{a}$, Rudy, V. ${ }^{a}$, Kanász, R. ${ }^{b}$, Gazda, V. ${ }^{\mathrm{c},}$ \\ ${ }^{a}$ Technical university of Kosice, Faculty of Mechanical Engineering; Institute of management, Industrial and Digital \\ Engineering, Slovak Republic \\ ${ }^{\mathrm{b}}$ Marwin Cassovia Soft, Ltd. \\ 'Technical university of Kosice, Faculty of Economics, Department of Finance, Slovak Republic
}

\section{A B S T R A C T}

The shortening of the production cycle and increasing impact of the technological innovations evokes improvement of the methods used in the production line scheduling. The aim of the presented research is a proposal of the decision model that enables a flexible reaction to the changing production conditions. The central decisions are substituted by the decisions performed on the independent machines level. The machines utilize rather restricted information on the capacities utilization of their technological neighbours. The decisions follow the decision tables (decision chromosomes) re-coded in the course of the evolution. The Genetic algorithms standing behind the model, enable identification of the highly acceptable solutions that is proved by a set of the simulation experiments. The set of independent machines becomes self-organized, having a significant positive effect on the production line capacities utilization. The decentralization aspect makes our proposal somewhat different from other research in the field. The philosophy is built on the fact that each machine makes its production decision based on the available information, which it has at its disposal in a given time. The machine flexible reacts to its neighbours' capacity utilization (machine before and after given production machine) in the production process flow.
\end{abstract}

\section{ARTICLE INFO}

Keywords:

Production line;

Job shop problem (JSP);

Decentralised optimization;

Production scheduling;

Shortest processing time rule;

Self-organization;

Genetic algorithm;

Decision table

*Corresponding author:

vladimir.gazda@tuke.sk

(Gazda, V.)

Article history:

Received 15 May 2020

Revised 16 October 2020

Accepted 19 October 2020

\section{References}

[1] Maccarthy, B.L., Liu, J. (1993). Addressing the gap in scheduling research: A review of optimization and heuristic methods in production scheduling, International Journal of Production Research, Vol. 31, No. 1, 59-79, doi: 10.1080/00207549308956713.

[2] Çaliş, B., Bulkan, S. (2015). A research survey: Review of AI solution strategies of job shop scheduling problem, Journal of Intelligent Manufacturing, Vol. 26, No. 5, 961-973, doi: 10.1007/s10845-013-0837-8.

[3] Nouiri, M., Bekrar, A., Jemai, A., Niar, S., Ammari, A.C. (2018). An effective and distributed particle swarm optimization algorithm for flexible job-shop scheduling problem, Journal of Intelligent Manufacturing, Vol. 29, No. 3, 603-615, doi: 10.1007/s10845-015-1039-3.

[4] Hoogeveen, H. (2005). Multicriteria scheduling, European Journal of Operational Research, Vol. 167, No. 3, 592623, doi: 10.1016/i.ejor.2004.07.011.

[5] Liu, J., Luo, X.-G., Zhang, X.-M., Zhang, F., Li, B.-N. (2013). Job scheduling model for cloud computing based on multi-objective genetic algorithm, International Journal of Computer Science Issues, Vol. 10, No. 1, 134-139.

[6] Zhang, G., Shao, X., Li, P., Gao, L. (2009). An effective hybrid particle swarm optimization algorithm for multiobjective flexible job-shop scheduling problem, Computers \& Industrial Engineering, Vol. 56, No. 4, 1309-1318, doi: 10.1016/i.cie.2008.07.021.

[7] Sonmez, A.I., Baykasoglu, A. (1998). A new dynamic programming formulation of $(\mathrm{n} \times \mathrm{m})$ flowshop sequencing problems with due dates, International Journal of Production Research, Vol. 36, No. 8, 2269-2283, doi: $\underline{10.1080 / 002075498192896 .}$. 
[8] Garey, M.R., Johnson, D.S., Sethi, R. (1976). The complexity of flowshop and jobshop scheduling, Mathematics of Operations Research, Vol. 1, No. 2, 117-129, doi: 10.1287/moor.1.2.117.

[9] Błażewicz, J., Domschke, W., Pesch, E. (1996). The job shop scheduling problem: Conventional and new solution techniques, European Journal of Operational Research, Vol. 93, No. 1, 1-33, doi: 10.1016/0377-2217(95)00362-2.

[10] Ouelhadj, D., Petrovic, S. (2009). A survey of dynamic scheduling in manufacturing systems, Journal of Scheduling, Vol. 12, No. 4, 417-431, doi: 10.1007/s10951-008-0090-8.

[11] Mohapatra, P., Nayak, A., Kumar, S.K., Tiwari, M.K. (2015). Multi-objective process planning and scheduling using controlled elitist non-dominated sorting genetic algorithm, International Journal of Production Research, Vol. 53, No. 6, 1712-1735, doi: 10.1080/00207543.2014.957872.

[12] Supsomboon, S., Vajasuvimon, A. (2016). Simulation model for job shop production process improvement in machine parts manufacturing, International Journal of Simulation Modelling, Vol. 15, No. 4, 611-622, doi: 10.2507/IJSIMM15(4)3.352.

[13] Ma, D.Y., He, C.H., Wang, S.Q., Han, X.M., Shi, X.H. (2018). Solving fuzzy flexible job shop scheduling problem based on fuzzy satisfaction rate and differential evolution, Advances in Production Engineering \& Management, Vol. 13, No. 1, 44-56, doi: 10.14743/apem2018.1.272.

[14] Ojstersek, R., Lalic, D., Buchmeister, B. (2019). A new method for mathematical and simulation modelling interactivity: A case study in flexible job shop scheduling, Advances in Production Engineering \& Management, Vol. 14, No. 4, 435-448, doi: 10.14743/apem2019.4.339.

[15] Shen, X.-N., Yao, X. (2015). Mathematical modeling and multi-objective evolutionary algorithms applied to dynamic flexible job shop scheduling problems, Information Sciences, Vol. 298, No. 20, 198-224, doi: 10.1016/ j.ins.2014.11.036.

[16] Meolic, R., Brezočnik, Z. (2018). Flexible job shop scheduling using zero-suppressed binary decision diagrams, Advances in Production Engineering \& Management, Vol. 13, No. 4, 373-388, doi: 10.14743/apem2018.4.297.

[17] Yu, M.R., Yang, B., Chen, Y. (2018). Dynamic integration of process planning and scheduling using a discrete particle swarm optimization algorithm, Advances in Production Engineering \& Management, Vol. 13, No. 3, 279-296, doi: 10.14743/apem2018.3.290.

[18] Holland, J.H. (1973). Genetic algorithms and the optimal allocation of trials, SIAM Journal on Computing, Vol. 2, No. 2, 88-105, doi: 10.1137/0202009.

[19] Holland, J.H., Reitman, J.S. (1978). Cognitive systems based on adaptive algorithms, Pattern-Directed Inference Systems, Vol. 1, No. 1, 313-329, doi: 10.1016/B978-0-12-737550-2.50020-8.

[20] Bierwirth, C., Kopfer, H., Mattfeld, D.C., Rixen, I. (1995). Genetic algorithm based scheduling in a dynamic manufacturing environment, In: Proceedings of the Second Conference on Evolutionary Computation, Perth, Australia, 439-443, doi: 10.1109/ICEC.1995.489188.

[21] Janes, G., Perinic, M., Jurkovic, Z. (2017). An efficient genetic algorithm for job shop scheduling problems, Tehnički Vjesnik - Technical Gazette, Vol. 24, No. 4, 1243-1247, doi: 10.17559/TV-20150527133957.

[22] Chen, W., Hao, Y.F. (2018). Genetic algorithm-based design and simulation of manufacturing flow shop scheduling, International Journal of Simulation Modelling, Vol. 17, No. 4, 702-711, doi: 10.2507/IJSIMM17(4)C017.

[23] Zhang, J., Ding, G., Zou, Y., Qin, S., Fu, J. (2019). Review of job shop scheduling research and its new perspectives under Industry 4.0, Journal of Intelligent Manufacturing, Vol. 30, 1809-1830, doi: 10.1007/s10845-017-1350-2.

[24] Oral, M., Malouin, J.-L. (1973). Evaluation of the shortest processing time scheduling rule with truncation process, AIIE Transactions, Vol. 5, No. 4, 357-365, doi: 10.1080/05695557308974923.

[25] Koza, J.R. (1992). Genetic programming: On the programming of computers by means of natural selection, MIT press, Cambridge, USA. 


\title{
Decentralizirana optimizacija fleksibilnih proizvodnih linij
}

\author{
Malega, P. ${ }^{a}$, Rudy, V. ${ }^{a}$, Kanász, R. ${ }^{b}$, Gazda, V. ${ }^{\text {, }}{ }^{*}$ \\ aTechnical university of Kosice, Faculty of Mechanical Engineering; Institute of management, Industrial and Digital \\ Engineering, Slovak Republic \\ bMarwin Cassovia Soft, Ltd. \\ 'Technical university of Kosice, Faculty of Economics, Department of Finance, Slovak Republic
}

\section{POVZETEK}

Skrajšanje proizvodnega cikla in vse večji vpliv tehnoloških inovacij povzročata izboljšanje metod, ki se uporabljajo pri terminiranju proizvodne linije. Cilj predstavljene raziskave je predlagati odločitveni model, ki omogoča fleksibilen odziv na spreminjajoče se proizvodne razmere. Osrednje odločitve so nadomeščene z odločitvami, ki se izvajajo na ravni neodvisnih strojev. Stroji uporabljajo precej omejene informacije o izkoriščenosti zmogljivosti svojih tehnoloških sosedov. Odločitve sledijo odločitvenim tabelam (odločitveni kromosomi), ki so bile na novo kodirane v teku evolucije. Genetski algoritem, ki stoji za modelom, omogoča identifikacijo zelo sprejemljivih rešitev, kar dokazuje niz simulacijskih poskusov. Skupina neodvisnih strojev se samoorganizira, kar pomembno pozitivno vpliva na izkoriščenost zmogljivosti proizvodne linije. Zaradi vidika decentralizacije se naš predlog nekoliko razlikuje od drugih raziskav na tem področju. Filozofija je zgrajena na tem, da vsak stroj svojo proizvodno odločitev sprejme na podlagi razpoložljivih informacij, s katerimi razpolaga $v$ danem času. Stroj se prilagodljivo odziva na izkoriščenost zmogljivosti svojih sosedov (stroj pred in po danem proizvodnem stroju) med proizvodnim procesom.

(C) 2020 CPE, University of Maribor. All rights reserved.

\begin{tabular}{l} 
P O D A T K I O Č L A N K U \\
\hline Ključne besede: \\
Proizvodna linija; \\
Težava proizvodnje po naročilu \\
(JSP); \\
Decentralizirana optimizacija; \\
Načrtovanje proizvodnje; \\
Pravilo najkrajšega časa obdelave; \\
Samoorganizacija; \\
Genetski algoritem; \\
Odločitvena tabela \\
*Kontaktna oseba: \\
vladimir.gazda@tuke.sk \\
(Gazda, V.) \\
Zgodovina članka: \\
Prejet 15. maja 2020 \\
Popravljen 16. oktobra 2020 \\
Sprejet 19. oktobra 2020
\end{tabular}

